\title{
A simplified proof of the Andre-Oort conjecture for products of modular curves
}

Article

Accepted Version

Daw, C. (2012) A simplified proof of the Andre-Oort conjecture for products of modular curves. Archiv der Mathematik, 98 (5). pp. 433-440. ISSN 1420-8938 doi:

https://doi.org/10.1007/s00013-012-0372-4 Available at https://centaur.reading.ac.uk/70357/

It is advisable to refer to the publisher's version if you intend to cite from the work. See Guidance on citing.

Published version at: http://link.springer.com/article/10.1007/s00013-012-0372-4

To link to this article DOI: http://dx.doi.org/10.1007/s00013-012-0372-4

Publisher: Springer

All outputs in CentAUR are protected by Intellectual Property Rights law, including copyright law. Copyright and IPR is retained by the creators or other copyright holders. Terms and conditions for use of this material are defined in the End User Agreement.

\section{www.reading.ac.uk/centaur}

\section{CentAUR}

Central Archive at the University of Reading

Reading's research outputs online 


\title{
A simplified proof of the André-Oort conjecture for prod- ucts of modular curves
}

\author{
Christopher Daw
}

\begin{abstract}
In this paper we give a short proof of the André-Oort conjecture for products of modular curves under the Generalised Riemann Hypothesis using only simple Galois-theoretic and geometric arguments. We believe this method represents a strategy for proving the conjecture for a general Shimura variety under GRH without using ergodic theory. We also demonstrate a short proof of the Manin-Mumford conjecture for Abelian varieties using similar arguments.
\end{abstract}

Mathematics Subject Classification (2010). Primary 11G18; Secondary 14G35, 11 G10.

Keywords. André-Oort, modular curve, Shimura variety, Manin-Mumford.

\section{Introduction}

This paper is concerned with the following conjecture.

Conjecture 1.1. (André-Oort) Let $S$ be a Shimura variety and let $\Sigma$ be a set of special points in $S$. Every irreducible component of the Zariski closure of $\Sigma$ is a special subvariety of $S$.

In this paper we consider the case when $S$ is a product of modular curves. However, since $S$ always admits a morphism $\pi$ to $\mathrm{SL}_{2}^{n}(\mathbb{Z}) \backslash \mathbb{H}^{n}$ and an irreducible subvariety $Z$ is special if and only if $\pi(Z)$ is special, we will assume $S$ to be $\mathrm{SL}_{2}^{n}(\mathbb{Z}) \backslash \mathbb{H}^{n}=\mathbb{C}^{n}$. Special subvarieties here have the following description (see [10], Definition 2.1).

Definition 1.2. Let $I=\{1, \ldots, n\}$. A closed irreducible subvariety $Z$ of $\mathbb{C}^{n}$ is called special (of type $\left.\Omega=\Omega_{Z}\right)$ if $I$ has a partition $\Omega=\left(I_{1}, \ldots, I_{t}\right)$, with $\left|I_{i}\right|=n_{i}$, such that $Z$ is a product of subvarieties $Z_{i}$ of $\mathbb{C}^{n_{i}}$, each of one of the following forms:

1. $I_{i}$ is a one element set and $Z_{i}$ is a CM point.

2. $Z_{i}$ is the image of $\mathbb{H}$ in $\mathbb{C}^{n_{i}}$ under the map sending $\tau$ in $\mathbb{H}$ to the image of $\left(g_{s} \cdot \tau\right)_{s \in I_{i}}$ in $\mathbb{C}^{n_{i}}$ for elements $g_{s} \in \mathrm{SL}_{2}(\mathbb{Q})$.

Given a special subvariety $Z$ of type $\Omega$, we define $c(\Omega)$ to be the number of CM factors. A special subvariety $Z$ is called strongly special if $c(\Omega)=0$.

The strategy of this paper will be to consider an irreducible subvariety $X$ in $\mathbb{C}^{n}$ containing a Zariski dense set $\Sigma$ of special subvarieties. After, if necessary, replacing $\Sigma$ by a Zariski dense subset, we may assume that $c\left(\Omega_{Z}\right)$ is constant as $Z$ ranges through $\Sigma$. Hence, we denote its value $c(\Sigma)$. Under GRH and using Galois-theoretic and geometric arguments, Ullmo and Yafaev show in [10] that, if $c(\Sigma)>0$, then $X$ contains a Zariski dense set $\Sigma^{\prime}$ with $c\left(\Sigma^{\prime}\right)=0$. Therefore, we consider sets of strongly special subvarieties. These are dealt with in [1] via ergodic theory. In this paper, we show that the irreducible components of the Zariski closure of a set of strongly special subvarieties are special using only simple geometric arguments. 
The result for products of modular curves has also been attained via relatively elementary methods by Edixhoven in [3]. However, his method relies intrinsically on the properties of products of modular curves. The motivation for this paper is the development of a strategy that will apply to a general Shimura variety. Using model theory, Pila has proven the André-Oort conjecture unconditionally for $\mathbb{C}^{n}$ (see [7]) and his ideas have led to unconditional proofs of other special cases e.g. $[2]$.

Given a set of strongly special subvarieties $\Sigma$, we consider an irreducible component $X$ of its Zariski closure. The idea of the proof is to intersect $X$ with its image under a suitable Hecke correspondence and reiterate this proceedure, each time with an irreducible component of the previous intersection. By comparing lower bounds for the degrees of strongly special subvarieties with the degrees of Hecke correspondences, we arrive at a nonproper intersection. For each $Z$ in $\Sigma$, we produce a $Z^{\prime}$ strictly containing $Z$ and repeat the argument.

\section{Degrees of strongly special subvarieties}

Consider the image of $\mathbb{H}$ in $\mathbb{C}^{n}$, for some $n \in \mathbb{N}$, under the map described in Definition 1.2 (2) for some $g_{1}, \ldots, g_{n} \in \mathrm{SL}_{2}(\mathbb{Q})$. For simplicity we may assume that $g_{1}$ is the identity. This image is the modular curve $\Gamma^{\prime} \backslash \mathbb{H}$ embedded in $\mathbb{C}^{n}$, with $\Gamma^{\prime}:=\Gamma \cap g_{2}^{-1} \Gamma g_{2} \cap \ldots \cap g_{n}^{-1} \Gamma g_{n}$, where $\Gamma:=\mathrm{SL}_{2}(\mathbb{Z})$.

By [5], Proposition 5.3.2., the projection

$$
\pi: \Gamma^{\prime} \backslash \mathbb{H} \rightarrow \Gamma \backslash \mathbb{H},
$$

extends to a morphism

$$
\bar{\pi}: \overline{\Gamma^{\prime} \backslash \mathbb{H}} \rightarrow \overline{\Gamma \backslash \mathbb{H}}
$$

of their Baily-Borel compactifications, such that the inverse image $\bar{\pi}^{*} L_{\Gamma}$ of the Baily-Borel line bundle on $\overline{\Gamma \backslash \mathbb{H}}$ is $L_{\Gamma^{\prime}}$, the Baily-Borel line bundle on $\overline{\Gamma^{\prime} \backslash \mathbb{H}}$, which is the restriction of the Baily-Borel line bundle on $\left(\mathbb{P}_{\mathbb{C}}^{1}\right)^{n}$. Therefore, by the projection formula, we have

$$
\operatorname{deg}_{L_{\mathbb{C}} n} \overline{\Gamma^{\prime} \backslash \mathbb{H}}=\operatorname{deg} \bar{\pi} \cdot \operatorname{deg}_{L_{\Gamma}} \overline{\Gamma \backslash \mathbb{H}}
$$

which is bounded below by the index $\left[\Gamma: \Gamma^{\prime}\right]$.

Consider the closures $\bar{\Gamma}=\mathrm{SL}_{2}(\hat{\mathbb{Z}})$ and $\overline{\Gamma^{\prime}}$ of $\Gamma$ and $\Gamma^{\prime}$ in $\mathrm{SL}_{2}(\hat{\mathbb{Z}})$, respectively. We have $\left[\Gamma: \Gamma^{\prime}\right] \geq$ $\left[\bar{\Gamma}: \overline{\Gamma^{\prime}}\right]$, which is equal to

$$
\prod_{\{p: \text { prime }\}}\left[\mathrm{SL}_{2}\left(\mathbb{Z}_{p}\right):\left(\mathrm{SL}_{2}\left(\mathbb{Z}_{p}\right) \cap g_{2}^{-1} \mathrm{SL}_{2}\left(\mathbb{Z}_{p}\right) g_{2} \cap \ldots \cap g_{n}^{-1} \mathrm{SL}_{2}\left(\mathbb{Z}_{p}\right) g_{n}\right)\right],
$$

noting that, for almost all $p, g_{i} \in \mathrm{SL}_{2}\left(\mathbb{Z}_{p}\right)$ for all $i=2, \ldots, n$. Therefore, the above quotients at these primes are trivial. Now suppose that $g_{i} \notin \mathrm{SL}_{2}\left(\mathbb{Z}_{p}\right)$ for some $i \in\{2, \ldots, n\}$ and a prime $p$. Considering Smith normal forms, it is possible to write $g_{i}=\gamma D \gamma^{\prime}$, where $\gamma, \gamma^{\prime} \in \mathrm{SL}_{2}\left(\mathbb{Z}_{p}\right)$ and $D$ is a diagonal matrix of the form $\operatorname{diag}\left(p^{n}, p^{-n}\right)$, for some $n \in \mathbb{N}$. Then

$$
\mathrm{SL}_{2}\left(\mathbb{Z}_{p}\right) \cap g_{2}^{-1} \mathrm{SL}_{2}\left(\mathbb{Z}_{p}\right) g_{2} \cap \ldots \cap g_{n}^{-1} \mathrm{SL}_{2}\left(\mathbb{Z}_{p}\right) g_{n}
$$

is contained in the subgroup of $\mathrm{SL}_{2}\left(\mathbb{Z}_{p}\right)$ whose lower left entry belongs to $p \mathbb{Z}_{p}$. The index of this subgroup can be calculated the same way as the index of $\Gamma_{0}(p)$ in $\mathrm{SL}_{2}(\mathbb{Z})$ (see [6], p81) and is bounded below by $p$.

Thus, from the above remarks, we conclude that the degree of $\Gamma^{\prime} \backslash \mathbb{H}$ with respect to the Baily Borel line bundle on $\left(\mathbb{P}_{\mathbb{C}}^{1}\right)^{n}$ is bounded below by the product of primes $p$ such that $g_{i} \notin \mathrm{SL}_{2}\left(\mathbb{Z}_{p}\right)$ for some $i \in\{2, \ldots, n\}$.

Henceforth, when we refer to the degree of a subvariety of $\mathbb{C}^{n}$, we will omit reference to the Baily Borel line bundle. 


\section{Choosing a suitable Hecke correspondence.}

Consider the Shimura variety $\operatorname{Sh}_{K}(G, X)_{\mathbb{C}}$ defined by the Shimura datum

$$
(G, X):=\left(\mathrm{GL}_{2, \mathbb{Q}}^{n},\left(\mathbb{H}^{ \pm}\right)^{n}\right)
$$

and $K:=\mathrm{GL}_{2}^{n}(\hat{\mathbb{Z}})$, a compact open subgroup of $\mathrm{GL}_{2}^{n}\left(\mathbb{A}_{f}\right)$; this is the connected Shimura variety $\mathbb{C}^{n}$. Consider $\mathrm{GL}_{2, \mathbb{Q}}$ emdedded into $\mathrm{GL}_{2, \mathbb{Q}}^{n}$ via the map

$$
\varphi: x \mapsto\left(x, g_{2} x g_{2}^{-1}, \ldots, g_{n} x g_{n}^{-1}\right),
$$

for $g_{2}, \ldots, g_{n} \in \mathrm{GL}_{2}(\mathbb{Q})$. We denote its image by $H$ and we write $X_{H}^{+}$for a connected component of $H(\mathbb{R}) \cdot\left(i, g_{2} i, \ldots, g_{n} i\right) \subset\left(\mathbb{H}^{ \pm}\right)^{n}$. Let $Z$ be the image of $X_{H}^{+} \times\{1\}$ in $\operatorname{Sh}_{K}(G, X)_{\mathbb{C}}$. This is the image of $\mathbb{H}$ described in the previous section.

Lemma 3.1. For any $\alpha^{2} \in H\left(\mathbb{A}_{f}\right), Z$ is contained in its image under the Hecke correspondence $T_{\alpha^{2}}$.

Proof. Let $\overline{(x, 1)} \in Z$ i.e. $x \in X_{H}^{+}$. Let $\tau \in \mathrm{GL}_{2}\left(\mathbb{A}_{f}\right)$ be such that $\tau=\varphi(\alpha)$. A point $\overline{(h, g)} \in$ $\operatorname{Sh}_{K}(G, X)_{\mathbb{C}}$ depends only on $g$ modulo $\left(\mathbb{A}_{f}^{*}\right)^{n}=\left(\mathbb{Q}^{*} \cdot \hat{\mathbb{Z}}^{*}\right)^{n}$, since these factors are killed in the double coset defining the Shimura variety. Hence, we consider the image of $\tau^{2}$ under the standard map

$$
\pi: \mathrm{GL}_{2, \mathbb{Q}} \rightarrow \mathrm{PGL}_{2, \mathbb{Q}},
$$

which is surjective on adelic points since the kernel $\mathbb{G}_{m, \mathbb{Q}}$ is connected. Consider the simply connected covering

$$
\rho: \mathrm{SL}_{2, \mathbb{Q}} \rightarrow \mathrm{PGL}_{2, \mathbb{Q}}
$$

Its kernel is $\mu_{2, \mathbb{Q}}$. Therefore, there exists a $v \in \mathrm{SL}_{2}\left(\mathbb{A}_{f}\right)$ such that $\rho(v)=\pi\left(\tau^{2}\right)$. By strong approximation applied to $\mathrm{SL}_{2, \mathbb{Q}}, v=q k$, with $q \in \mathrm{SL}_{2}(\mathbb{Q})$ and $k$ belonging to the compact open subgroup

$$
\bigcap_{i=1}^{n} g_{i}^{-1} \mathrm{SL}_{2}(\hat{\mathbb{Z}}) g_{i} \subset \mathrm{SL}_{2}\left(\mathbb{A}_{f}\right),
$$

where $g_{1}=1$. Note that $\pi$ restricted to $\mathrm{SL}_{2, \mathbb{Q}}$ coincides with $\rho$, since $\rho$ is a universal cover. Now, $\varphi(q)$ belongs to $H(\mathbb{Q})^{+}$. Hence, $\varphi(q) \cdot x$ belongs to $X_{H}^{+}$. Therefore, consider $\overline{\left(\varphi(q) \cdot x, \varphi\left(\tau^{2}\right)\right)} \in T_{\alpha^{2}}(Z)$. By the previous discussion, this equals $\overline{(\varphi(q) \cdot x, \varphi(q k))}=\overline{(x, \varphi(k))}$. However, since $\varphi(k) \in \mathrm{GL}_{2}^{n}(\hat{\mathbb{Z}})$, this point is $\overline{(x, 1)}$.

Choose a prime $p$ such that $g_{i} \in \mathrm{GL}_{2}\left(\mathbb{Z}_{p}\right)$ for all $i=2, \ldots, n$. Consider the element

$$
P:=\left(\begin{array}{cc}
1 & 0 \\
0 & p^{-2}
\end{array}\right) \in \mathrm{GL}_{2}(\mathbb{Q})^{+}
$$

Let $\alpha \in H\left(\mathbb{Q}_{p}\right)$ be the image of $P$ in $\mathrm{GL}_{2}^{n}\left(\mathbb{Q}_{p}\right)$ under $\varphi$. Since the $g_{i}$ belong to $\mathrm{GL}_{2}\left(\mathbb{Z}_{p}\right)$ for all $i=2, \ldots, n$, the double coset $K \alpha K$ equals $K \beta K$, where $\beta$ is $P$ diagonally embedded into $\mathrm{GL}_{2}^{n}\left(\mathbb{Q}_{p}\right)$.

Recall that $\left([4]\right.$, Theorem 6.1) the connected components of the correspondence $T_{\beta}$ on $\mathbb{C}^{n}$ are the $T_{\beta_{i}}$ induced by $\mathrm{GL}_{2}^{n}(\mathbb{Q})^{+}$acting on $\mathbb{H}^{n}$ such that

$$
\mathrm{GL}_{2}^{n}(\mathbb{Q})^{+} \cap K \beta K=\coprod_{i} \Gamma^{n} \beta_{i}^{-1} \Gamma^{n} .
$$

Note, however, that $\mathrm{GL}_{2}^{n}(\mathbb{Q})^{+} \cap K \beta K=\Gamma^{n} \beta \Gamma^{n}$. Hence, the correspondence $T_{\beta}$ is irreducible and equal to the standard Hecke correspondence $T_{p^{2}}$ on $\mathbb{C}^{n}$. Combining this observation with lemma 3.1, we obtain the following result:

Lemma 3.2. Let $Z$ be the image of $\mathbb{H}$ in $\mathbb{C}^{n}$ as defined above. Choose a prime $p$ such that $g_{i} \in \mathrm{SL}_{2}\left(\mathbb{Z}_{p}\right)$ for all $i=2, \ldots, n$. Then $Z$ is contained in its image under the Hecke correspondence $T_{p^{2}}$ on $\mathbb{C}^{n}$ given by $P^{-1}$. 


\section{Proof}

Theorem 4.1. Assume the GRH for imaginary quadratic fields. Let $X$ be an irreducible component of the Zariski closure of a set $\Sigma$ of special subvarieties in a product $S$ of $n$ modular curves. Then $X$ is a special subvariety.

By [10] we may assume that $c(\Sigma)=0$ i.e. $\Sigma$ is a set of strongly special subvarieties. The GRH will not be used in this case. Using Proposition 2.1 of [4] we may assume that $S$ is the product of $n$ copies of $\mathrm{SL}_{2}(\mathbb{Z}) \backslash \mathbb{H}$.

Proof. Consider a special subvariety $Z \subset X \subset \mathbb{C}^{n}$. Given our description of strongly special subvarieties we write $Z=Z_{1} \times \cdots \times Z_{t}$, where each $Z_{i}$ is the image of $\mathbb{H}$ in $\mathbb{C}^{n_{i}}$ given by some $g_{i_{2}}, \ldots, g_{i_{n_{i}}} \in \mathrm{SL}_{2}(\mathbb{Q})$. Therefore, by the arguments in Section 2 , the degree of $Z$ is bounded below by the product of all primes $p$ such that not all $g_{i_{j}} \in \mathrm{SL}_{2}\left(\mathbb{Z}_{p}\right)$ for $i=1, \ldots, t$ and $j=2, \ldots, n_{i}$. We denote this product $M_{Z} \in \mathbb{N}$. By Lemma 3.2, for any prime $p$ not dividing $M_{Z}$, we have $Z \subset T_{p^{2}}(Z)$,

By [3], Section 3, we may assume that $X$ is a hypersurface all of whose projections to any $n-1$ factors of $\mathbb{C}^{n}$ are dominant. Under this assumption, given $n \geq 3, T_{p^{2}}(X)$ is irreducible for any $p$ greater than $\operatorname{deg} X$ and 13 by Proposition 3.1 of [10]. If $n=2, Z$ is either $\mathbb{C}^{2}$ or a modular curve $Y_{0}(N)$, in which case $X=\mathbb{C}^{2}$ or $X=Y_{0}(N)$.

Consider first the case that $X$ contains a Zariski dense subset $\Sigma^{\prime}$ such that $M_{Z}$ is bounded for all $Z$ in $\Sigma^{\prime}$ i.e. the elements $g_{i_{j}}$ defining a given $Z$ must belong to $\mathrm{SL}_{2}\left(\mathbb{Z}_{p}\right)$ for all but a fixed and finite set of primes whose product we denote $M$. For any prime $p$ not dividing $M$, every $Z$ in $\Sigma^{\prime}$ is contained in its image under $T_{p^{2}}$. Hence, $\Sigma^{\prime}$ belongs to $X \cap T_{p^{2}}(X)$. This is a closed set and so $X \subset T_{p^{2}}(X)$. However, since $T_{p^{2}}(X)$ is irreducible (provided $p$ is larger than a uniform constant), we must have

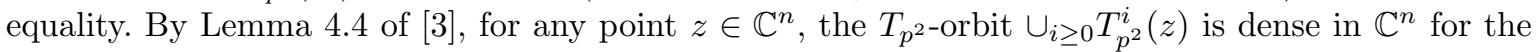
Archimedean topology. Thus, $X$ is $\mathbb{C}^{n}$.

Therefore, we assume that $X$ contains no such Zariski dense subset. Hence, we may assume that $M_{Z}$ is larger than any uniform constant for all $Z$ in $\Sigma$. We consider an arbitrary $Z$. By a theorem of Chebyshev, there exist positive absolute constants $c_{1}$ and $c_{2}$ such that the number of primes less than a given real number $x \geq 2, \pi(x)$, is bounded below by $c_{1} \frac{x}{\log x}$ and above by $c_{2} \frac{x}{\log x}$. Therefore, for any fixed $0<\delta_{1}<1$ and $\epsilon>0$,

$$
\pi\left(M_{Z}^{\delta_{1}}\right) \gg \frac{M_{Z}^{\delta_{1}}}{\log M_{Z}^{\delta_{1}}} \gg M_{Z}^{\delta_{1}-\epsilon} .
$$

It is an obvious fact that the number of primes dividing $M_{Z}, \omega\left(M_{Z}\right)$, satisfies

$$
\omega\left(M_{Z}\right) \leq \frac{\log M_{Z}}{\log 2} \ll M_{Z}^{\epsilon}
$$

Therefore, for $M_{Z}$ larger than a constant depending only on $X$, we can find a prime $p_{1}$ smaller than $M_{Z}^{\delta_{1}}$, not dividing $M_{Z}$ and larger than 3 and the degree of $X$. The last condition implies that $T_{p_{1}^{2}}(X)$ is irreducible. Therefore, the intersection of $X$ and $T_{p_{1}^{2}}(X)$ is either proper or $X=T_{p_{1}^{2}}(X)$. If $X=T_{p_{1}^{2}}(X)$ then we are finished, as explained above.

Thus, we assume the intersection is proper. For the prime $p_{1}, Z \subset T_{p_{1}^{2}}(Z)$, which implies $Z \subset$ $X \cap T_{p_{1}^{2}}(X)$. We relabel $X$ as $X_{1}$ and let $X_{2}$ be an irreducible component of the intersection containing $Z$. Notice that, by Bezout's theorem ([9], Lemme 3.4), the degree of $X_{2} \subset X_{1} \cap T_{p_{1}^{2}}\left(X_{1}\right)$ is bounded above by $\left(\operatorname{deg} X_{1}\right)^{2} \cdot \operatorname{deg} T_{p_{1}^{2}}$, where

$$
\operatorname{deg} T_{p_{1}^{2}}=\left|\Gamma: \Gamma_{0}\left(p_{1}^{2}\right)\right|^{n}=p_{1}^{n}\left(p_{1}+1\right)^{n} \ll p_{1}^{2 n},
$$

i.e. $\operatorname{deg} X_{2} \ll p_{1}^{2+2 n} \ll M_{Z}^{(2+2 n) \delta_{1}}$. 
So long as $\delta_{1}$ and $\epsilon$ are small enough, we can fix $0<\delta_{2}<1$ such that $(2+2 n) \delta_{1}+2 \epsilon<\delta_{2}$. We have

$$
\pi\left(M_{Z}^{(2+2 n) \delta_{1}}\right) \ll \frac{M_{Z}^{(2+2 n) \delta_{1}}}{\log M_{Z}^{(2+2 n) \delta_{1}}} \ll M_{Z}^{(2+2 n) \delta_{1}+\epsilon}
$$

and

$$
\pi\left(M_{Z}^{\delta_{2}}\right) \gg \frac{M_{Z}^{\delta_{2}}}{\log M_{Z}^{\delta_{2}}} \gg M_{Z}^{\delta_{2}-\epsilon} .
$$

Therefore, for $M_{Z}$ larger than a constant depending only on $X$, we can a find a prime $p_{2}$ smaller than $M_{Z}^{\delta_{2}}$, not dividing $M_{Z}$ and larger than $\operatorname{deg} X_{2}$ and 3 . The second condition implies that $Z \subset X_{2} \cap T_{p_{2}^{2}}\left(X_{2}\right)$. The latter condition implies that if this interesection is not proper i.e. $X_{2} \subset T_{p_{2}^{2}}\left(X_{2}\right)$, then $X_{2}$ is special by [3], Theorem 4.1. It can only be strongly special since it contains $Z$. It is also of higher dimension than $Z$ by comparing degrees. Hence, we replace $Z$ in $\Sigma$ with $X_{2}$.

Assume, then, that the intersection is proper. We perform the above construction recursively, with suitable $\delta_{k}$, assuming $M_{Z}$ is large enough, thus obtaining subvarieties $X_{k}$ and primes $p_{k}$. If at some point we have $X_{k} \subset T_{p_{k}^{2}}\left(X_{k}\right), X_{k}$ is a special subvariety containing $Z$. If this inclusion does not occur at any of the previous stages, after a finite number of steps bounded by $\operatorname{dim} X_{1}$, we end up in the following situation:

1. $\operatorname{dim}\left(X_{k}\right)=\operatorname{dim}(Z)+1$

2. $\operatorname{deg}\left(X_{k}\right) \ll M_{Z}^{(2+2 n) \delta_{k-1}}$

3. $\operatorname{deg} Z>M_{Z}$

4. $Z \subset X_{k}$

5. $p_{k}<M_{Z}^{\delta_{k}}$

6. $p_{k}$ not divinding $M_{Z}$

7. $p_{k}>\operatorname{deg}\left(X_{k}\right)$

Therefore, we have $Z \subset X_{k} \cap T_{p_{k}^{2}}\left(X_{k}\right)$. By comparing the degrees of $Z$ and $X_{k} \cap T_{p_{k}^{2}}\left(X_{k}\right)$, the intersection cannot be proper. Hence, $X_{k}$ is contained in $T_{p_{k}^{2}}\left(X_{k}\right)$ and is, therefore, special by [3], Theorem 4.1.

We perform this procedure on all of the $Z$, replacing them in $\Sigma$ by strongly special subvarieties of higher dimension. Reiterating the above argument, we eventually conclude that $X$ must be special.

\section{Manin-Mumford}

We conclude this note by remarking that the techniques exhibited above apply, in a simpler way, to the Manin-Mumford conjecture for Abelian varieties.

Theorem 5.1. (Manin-Mumford) Let $K$ be a number field, $A / K$ an Abelian variety over $K$ and $V / K$ a geometrically irreducible subvariety of $A$. If $V(\bar{K})$ contains a Zariski dense set of torsion points then $V$ is the translate of an Abelian subvariety by a torsion point.

Many proofs of this theorem exist. Ratazzi and Ullmo have recently given another combining Galois-theoretic and ergodic methods. We refer to their paper [9] for more details. Here we replace the ergodic theory with an elementary geometric argument. In this setting, special subvarieties are the translates of Abelian subvarieties by torsion points.

Proof. Let $\left(\Sigma_{n}\right)_{n \in \mathbb{N}}$ be a sequence of special subvarieties with Zariski closure equal to $V$. For each $n \in \mathbb{N}$ we choose a representation

$$
\Sigma_{n}=A_{n}+\xi_{n}
$$


where $A_{n} \subset A$ is an Abelian subvariety and $\xi_{n}$ is a torsion point in the Abelian subvariety $A_{n}^{\prime}$ such that $A=A_{n}+A_{n}^{\prime}$ and $A_{n} \cap A_{n}^{\prime}$ is finite of uniformly bounded order (see [9], Proposition 2.1). Let $d_{n}$ denote the order of the torsion point $\xi_{n}$.

Whether or not the sequence $\left(d_{n}\right)_{n \in \mathbb{N}}$ is bounded is independent of the choice of the $\xi_{n}([9]$, Remarque 3.1). In the case that the sequence $\left(d_{n}\right)_{n \in \mathbb{N}}$ is unbounded, Section 3.2 of [9] concludes that each $\Sigma_{n}$ is contained in a special subvariety $\Sigma_{n}^{\prime}$ of higher dimension (the arguments here are Galoistheoretic, similar to the Shimura case, but are not dependent on GRH). Therefore, we replace $\left(\Sigma_{n}\right)_{n \in \mathbb{N}}$ with $\left(\Sigma_{n}^{\prime}\right)_{n \in \mathbb{N}}$ and reiterate this argument unless, at some point, we obtain a sequence $\left(\Sigma_{n}\right)_{n \in \mathbb{N}}$ with $\left(d_{n}\right)_{n \in \mathbb{N}}$ bounded.

In this case, since the set of torsion points of bounded order is finite, we may suppose that each $\Sigma_{n}$ is of the form $A_{n}+\xi$, for a fixed torsion point $\xi$. However, since $V$ is special if and only if $V-\xi$ is special, we may assume that $\Sigma_{n}=A_{n}$ for all $n \in \mathbb{N}$.

We denote by $[m]$ the multiplication by $m \in \mathbb{N}$ map on $A$ and choose one of the $A_{n}$. Consider the intersection $V \cap[m] V$. Either it is proper or $[m] V=V$, in which case $V$ is special by [9], Lemme 3.2. Notice that, by Bezout's theorem, the degree of $V \cap[m] V$ is bounded above by $\operatorname{deg} V \cdot \operatorname{deg}[m] V$ where, by [9], Lemme 3.1,

$$
\operatorname{deg}[m] V \leq m^{2 \operatorname{dim} V} \cdot \operatorname{deg} V .
$$

It is a classical fact that an Abelian variety contains only finitely many Abelian subvarieties of bounded degree. Therefore, we may assume that the $A_{n}$ have degree exceeding any uniform constant. Therefore, by comparing degrees, if $\operatorname{dim} V=\operatorname{dim} A_{n}+1$ and $\operatorname{deg} A_{n}>m^{2 \operatorname{dim} V} \cdot(\operatorname{deg} V)^{2}$, we must have $V=[m] V$.

If the intersection is proper we choose an irreducible component $\mathrm{W}$ of the intersection containing $A_{n}$. Therefore, $A_{n}$ is contained in the intersection $W \cap[m] W$. Either this intersection is proper or $W=[m] W$ and $W$ is special, in which case we replace $A_{n}$ in $\left(\Sigma_{n}\right)_{n \in \mathbb{N}}$ by $W$, a special subvariety of higher dimension. Again, comparing degrees, if $\operatorname{dim} W=\operatorname{dim} A_{n}+1$ and $\operatorname{deg} A_{n}>m^{8 \operatorname{dim} V} \cdot(\operatorname{deg} V)^{4}$, we must have $W=[m] W$.

Otherwise, if $W \cap[m] W$ is a proper intersection, we take an irreducible component containing $A_{n}$ and repeat the argument in the previous paragraph. After a finite number of steps, bounded by $\operatorname{dim} V$, we will have found a special subvariety strictly containing $A_{n}$. We perform this procedure on all of the $A_{n}$, replacing them by special subvarieties of $V$ of higher dimension. Reiterating the above arguments at most $\operatorname{dim} V-1$ times, we conclude that $V$ must be special.

\section{Acknowledgment}

The author would like to express his continuing gratitude to Andrei Yafaev for the abundance of ideas and time that he is willing to share. The author is also grateful to the department of mathematics at UCL for their financial support and to the referee for highlighting a mistake in the first version of this paper. 


\section{Bibliography.}

[1] Clozel, L and Ullmo, E (2005), Equidistribution de sous-variétés spéciales, Annals of Maths, 161, 1571-1588

[2] Daw, C and Yafaev, A (2011), An unconditional proof of the André-Oort conjecture for Hilbert modular surfaces, Manuscripta Math. 135, 263-271

[3] Edixhoven, B (2005), Special points on products of modular curves, Duke Mathematical Journal. Vol. 126. No. 2

[4] Edixhoven, S.J. and Yafaev, A (2003), Subvarieties of Shimura varieties, Annals of Mathematics, Volume 157, No. 2, March 2003, 621-645

[5] Klingler, B and Yafaev, A (2006), The André-Oort conjecture, Preprint

[6] Milne, J.S. (2009), Modular Functions and Modular Forms, available on the author's web page

[7] Pila, J (2011), O-minimality and the Andre-Oort conjecture for $\mathbb{C}^{n}$, Annals Math. 173, 1779-184

[8] Platanov, V. P. and Rapinchuk, A. S. (1991), Algebraic Groups and Number Theory, Academic Press, Inc.

[9] Ratazzi, N and Ullmo, E (2009), Galois+Equidistribution=Manin-Mumford, Ecole d't Arithmetic geometry, 419-430, Clay Math. Proc., 8, Amer. Math. Soc., Providence, RI

[10] Ullmo, E and Yafaev, A (2009), The André-Oort conjecture for products of modular curves, Arithmetic Geometry, 431-439, Clay Math. Proc., 8, Amer. Math. Soc., Providence, RI

[11] Ullmo, E and Yafaev, A (2006), Galois Orbits and equidistribution of special subvarieties: towards the André-Oort conjecture, with an appendix by P. Gille and L. Moret-Bailly, Preprint

Christopher Daw

College London

Department of Mathematics

Gower street

WC1E 6BT London

United Kingdom

e-mail: c.daw@ucl.ac.uk 